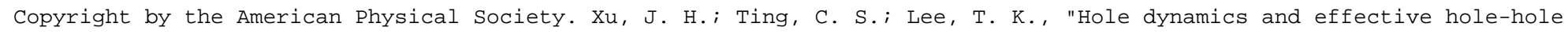

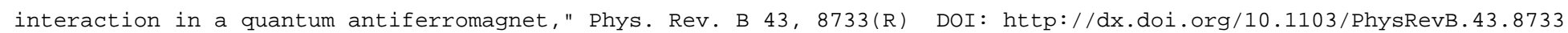

\title{
Hole dynamics and effective hole-hole interaction in a quantum antiferromagnet
}

\author{
J. H. Xu and C. S. Ting \\ Department of Physics and Texas Center for Superconductivity, University of Houston, Houston, Texas 77204 \\ T. K. Lee \\ Department of Physics, Virginia Polytechnic Institute and State University, Blacksburg, Virginia 24061
}

(Received 2 November 1990)

\begin{abstract}
By the use of an effective Hamiltonian which takes into account the constraint on the motion of holes in a quantum antiferromagnet, the dynamics of holes is investigated. It is found that there is a quasiparticle band whose width is of order $J$ at the bottom of the hole spectrum. The effective hole-hole interaction mediated by spin fluctuations is derived and the attraction between holes in the $d$-wave channel is obtained. The results suggest that a $d$-wave-pairing condensation in a quantum antiferromagnet is very possible.
\end{abstract}

The discovery of high-temperature superconductors has led to a revival of interest in two-dimensional (2D) strongly correlated electron systems. Anderson ${ }^{1}$ has suggested that the physics of these materials is contained in a $2 \mathrm{D}$, large- $U$, single-band Hubbard model. In the large- $U$ limit, the Hubbard model can be transformed into the $t-J$ model Hamiltonian ${ }^{2}$

$$
\begin{aligned}
H=H_{t}+H_{J}= & -t \sum_{\langle i j\rangle \sigma}\left[\left(1-n_{i-\sigma}\right) C_{i \sigma}^{\dagger} C_{j \sigma}\left(1-n_{j-\sigma}\right)+\text { H.c. }\right] \\
& +J \sum_{\langle i j\rangle} \mathbf{S}_{i} \cdot \mathbf{S}_{j},
\end{aligned}
$$

where $n_{i \sigma}=C_{i \sigma}^{\dagger} C_{i \sigma}$. The $C_{i \sigma}^{\dagger}$ are the usual electroncreation operators and the rest of the notation is standard. At half-filling, Hamiltonian (1) reduces to the antiferromagnetic Heisenberg model $H_{J}$ which develops longrange Néel order at zero temperature.

Away from half filling, in order to study the dynamics of holes in a doped antiferromagnet (AF), numerous approximation methods ${ }^{3-8}$ have been proposed. They revealed that the characteristic energy describing the coherent motion of the quasiparticle holes is $J$ and not $t$ (for $t>J$ ). The reason is that the hole motion is strongly coupled to the spin dynamics. Above the quasiparticle band there exists an incoherent band whose width is of order $t$. The possible existence of a Fermi surface for the holes is currently actively debated. In particular, the locations of the hole-band minimum are of general concern. Two possibilities have been considered so far: the hole could be located in momentum space (i) at $(\pi / 2, \pi / 2)$ or (ii) in the corners of the square defining the pseudo-Fermi surface, i.e., $(\pi, 0)$ or $(0, \pi)$. Such a result would imply that at low temperatures when holes are added to the system they will populate the region near that momentum, thus creating a pocketlike Fermi surface. Recent numerical studies for small clusters ${ }^{9,10}$ do not seem to find evidence of pockets in the Fermi surface. These interesting results may indicate that the difference in energy between $(\pi / 2, \pi / 2)$ and $(0, \pi)$ is very small, i.e., the energy of a hole at these different momenta is almost degenerate. Thus, with respect to a possible location of the band minimum for an antiferromagnetic (AF) system with one hole, the results from different approaches have failed to arrive at a definite conclusion.

Recently, Dagotto, Riera, and Young ${ }^{11}$ calculated numerically the dynamical-pairing susceptibility predicting a large low-energy spectral weight of the pair-pair correlation function in the $d$-wave channel, which suggests that the ground state of holes more likely behaves like a quasiparticle state at $(\pi, 0)$ or $(0, \pi)$ rather than at $(\pi / 2, \pi / 2)$. Very recently, Poilblanc and Dagotto ${ }^{12}$ investigated the spectral function by exact diagonalization of a small cluster with two holes; they found indications of a holequasiparticle state with minimum energy at $(\pi, 0)$ and $(0, \pi)$ momenta, in agreement with pairing-susceptibility results. ${ }^{10,11}$ Here we wish to address this question within the framework of an effective-Hamiltonian approach. We shall show that a hole in the 2D AF background can be described by a narrow quasiparticle band whose width is of order $J$. The band minimum of the single hole seems to be located in the corners of the pseudo-Fermi surface, but the energy at $(\pi, 0)$ and $(\pi / 2, \pi / 2)$ is almost degenerate. In addition, we also derive the effective interaction between holes mediated by the spin fluctuations and find that the quasiparticle-pair interaction is always attractive in the $d$-wave channel. We claim that the $d$-wave superconductivity in the system is consistent with both the quasiparticle property and the type of the pair potential. The present results are consistent with the recent numerical calculations. ${ }^{10-12}$

Let us look at the first term $H_{t}$ in Eq. (1) that describes the hopping of holes from site to site under the constraint that no double occupancy of sites is allowed. Although different analytical methods have been presented, ${ }^{5-8}$ the difficulty associated with the treatment of the constraint still remains to be resolved. Here we will give a different treatment to the hopping Hamiltonian $H_{t}$. We note from Eq. (1) that $H_{t}$ can be rewritten exactly in the following form:

$H_{t}=-t \sum_{\langle i j\rangle}\left(S_{i}^{+} C_{i \uparrow}^{\dagger} C_{j \downarrow} S_{j}^{-}+S_{i}^{-} C_{i \dagger}^{\dagger} C_{j \uparrow} S_{j}^{+}+\right.$H.c. $)$,

where $S_{i}^{+}=C_{i \dagger}^{\dagger} C_{i \downarrow}$ and $S_{i}^{-}=C_{i \dagger}^{\dagger} C_{i \uparrow}$. Now there is a different interpration for $H_{t}$ in the above equation: the 
holes in an AF background cannot move freely but couple strongly with the spin background, i.e., the motion of the holes in an AF background is always accompanied with absorption and emission of spin waves. So, $H_{t}$ in Eq. (2) can be regarded as properly describing the alteration of spin configurations as the hole moves. To elucidate this point clearly, in the following, we shall employ a crucial approximation to the spin operators $S_{i}^{ \pm}$and $S_{j}^{ \pm}$in Eq. (2), i.e., we replace them by the bosonic spin-wave operators $a_{i}$ and $a_{j}$ defined on each sublattice through the usual Holstein-Primakoff transformation. We now rewrite $H_{t}$ in Eq. (2) in terms of spin-wave operators and keep only the leading terms in $1 / S$, the Hamiltonian $H_{t}$ becomes

$$
H_{t}=-t \sum_{\langle i j\rangle}\left(C_{i \dagger}^{\dagger} C_{j \uparrow} a_{i}^{\dagger} a_{j}^{\dagger}+C_{i \dagger}^{\dagger} C_{j \downarrow} a_{i} a_{j}+\text { H.c. }\right),
$$

where the factor of $2 S$ is absorbed into $t$. Comparing with the original Hamiltonian (1), $H_{t}$ in Eq. (3) should act on the space with no doubly occupied sites, which can be established by introducing a constraint:

$$
2 a_{i}^{\dagger} a_{i}+\left(1-\sum_{\sigma} C_{i \sigma}^{\dagger} C_{i \sigma}\right)=2 S \text {. }
$$

Equations (3) and (4) describe both the hole hopping and hole interaction with the spin background.

At half filling (when there are no free fermions) and because $\sum_{\sigma} C_{i \sigma}^{\dagger} C_{i \sigma}=1$, the problem is then to solve the Heisenberg model $H_{J}$ under the constraint $S_{z}=S-a_{i}^{\dagger} a_{i}$ $=0$, which is equivalent to Takahashi's and Hirsch and Tang's recent modified spin-wave (MSW) theory for the 2D antiferromagnet, ${ }^{13,14}$ and closely related to the Schwinger-boson theory. ${ }^{15,16}$ Based upon the MSW theory, diagonalization of $H_{J}$ yields the following results: ${ }^{13,14}$

$$
\begin{aligned}
& H_{J}=\text { const }+\sum_{\mathbf{k}} \omega_{k} \alpha_{k}^{\dagger} \alpha_{k}, \\
& \omega_{\mathbf{k}}=\frac{z J S}{\eta}\left(1-\eta^{2} \gamma_{k}^{2}\right)^{1 / 2},
\end{aligned}
$$

with $\gamma_{\mathbf{k}}=\Sigma_{\delta} \exp (i \mathbf{k} \cdot \delta) / z$, where $\delta$ are the vectors to the $z$ nearest-neighbor sites. $\eta=1 /(1+\mu / 2 z S J)$, and $\mu$ is the Lagrange multiplier determined by the constraint. $\alpha$ is the spin-wave operator, $\alpha_{k}=a_{k} \cosh \theta_{k}+a_{-k}^{\dagger} \sinh \theta_{k}$ with $\tanh 2 \theta_{\mathbf{k}}=\eta \gamma_{\mathbf{k}}$. For the 2D square Heisenberg model, both the MSW and the conventional spin-wave theories give a ground state with long-range Néel order. For finite temperatures, the MSW theory can give a gap in the spin-excitation spectrum, which is consistent with the Monte Carlo calculations ${ }^{17}$ and renormalization-group theory. ${ }^{18}$

Next, we consider the hole dynamics. If holes are added to the system, we can see from Eq. (3) that each hole couples with two spin waves when it hops. The mean-field theory will yield a bare-hopping term in which holes can hop from one site to another without changing the spin configuration. Replacing $\mathrm{C}^{\dagger} \mathrm{Ca}^{\dagger} a^{\dagger}$ in Eq. (3) by $C^{\dagger} C\left\langle a^{\dagger} a^{\dagger}\right\rangle+\left\langle C^{\dagger} C\right\rangle a^{\dagger} a^{\dagger}$, and similarly for the other terms, the bare hopping term can be easily obtained from a mean-field theory:

$$
\varepsilon_{\mathrm{k}}^{B}=-z t \sum_{\mathrm{p}} \gamma_{\mathrm{k}-\mathrm{p}} \sinh 2 \theta_{\mathrm{p}} \approx-0.58 z t \gamma_{\mathrm{k}} \text {. }
$$

Based on this mean-field theory we would expect a hole bandwidth of order $t$. Indeed, the reason that this solution is favored in the mean-field theory is that it has the large bare hopping term so that the hole may acquire a large kinetic energy. The Schwinger-boson approach based upon the mean-field theory ${ }^{19,20}$ also predicted a similar result that there exists a coherent hole band whose width is of order $t$. However, since the holes are strongly coupled to the spin waves, it is important to consider the quasiparticle holes, which are dressed by a cloud of spin excitations. The true hole propagator $G(i, j, \omega)$ is defined by

$$
G(i, j, \omega)=\int_{-\infty}^{\infty} d t e^{i \omega t}\left\langle T\left[C_{i \sigma}^{\dagger}(t) C_{j \sigma}(0)\right]\right\rangle
$$

We consider a self-consistent perturbation theory in which vertex correlations are ignored. At zero temperature, the self-consistent integral equation for the hole propagator is given by

$$
G(\mathbf{k}, \omega)=\frac{1}{\omega-\varepsilon_{\mathbf{k}}^{B}-z^{2} t^{2} \sum_{\mathbf{p}, \mathbf{q}} \gamma_{\mathbf{k}}^{2}-\mathbf{p} f(\mathbf{p}, \mathbf{q}) G\left(\mathbf{k}-\mathbf{q}, \omega-\omega_{\mathbf{p}}-\omega_{\mathbf{p}-\mathbf{q}}\right)},
$$

where

$$
f(\mathbf{p}, \mathbf{q})=\left(\cosh \theta_{\mathbf{p}} \cosh \theta_{\mathbf{p}-\mathbf{q}}-\sinh \theta_{\mathbf{p}} \sinh \theta_{\mathbf{p}-\mathbf{q}}\right)^{2} .
$$

The dispersion of the quasiparticle band is given by

$$
\varepsilon_{\mathbf{k}}=\varepsilon_{\mathbf{k}}^{B}+z^{2} t^{2} \sum_{\mathbf{p}, \mathbf{q}} \gamma_{\mathbf{k}-\mathbf{p}}^{2} f(\mathbf{p}, \mathbf{q}) G\left(\mathbf{k}-\mathbf{q}, \varepsilon_{\mathbf{k}}-\omega_{\mathbf{p}}-\omega_{\mathbf{p}-\mathbf{q}}\right)
$$

The qualitative information about the low-energy structure of the hole spectrum can be obtained by using the dominantpole approximation. ${ }^{6}$ In this case, we may write the mass at the bottom of the band as

$$
\frac{1}{m_{i j}}=\frac{\partial^{2}}{\partial k_{i} \partial k_{j}} \varepsilon_{\mathbf{k}}=R_{\mathbf{k}} \frac{\partial^{2}}{\partial k_{i} \partial k_{j}}\left(\varepsilon_{\mathbf{k}}^{B}+z^{2} t^{2} \sum_{\mathbf{p}, \mathbf{q}} \gamma_{\mathbf{k}-\mathbf{p}}^{2} f(\mathbf{p}, \mathbf{k}-\mathbf{q}) G^{0}\left(\mathbf{q}, \omega_{0}\right)\right),
$$

where $R_{\mathrm{k}}$ is the quasiparticle residue, and $G^{0}\left(\mathbf{q}, \omega_{0}\right) \approx-1 / t$ is the $J=0$ limit with band edge at $\omega_{0}$. Employing the dominant pole approximation we found that $R_{\mathrm{k}}$ is of order $J / t$. Thus, both the bare-hopping term and the self-energy term are renormalized by the quasiparticle residue, leading to an effective mass which is enhanced by $t / J$. Under the effective-mass approximation, we conclude that the dispersion of the quasiparticle $\varepsilon_{k} \sim J$ near the bottom of the band. In the $J=0$ limit, $R_{\mathrm{k}}=0$ and the hole spectrum becomes completely incoherent. 
The position of the band minimum can be identified from Eq. (12). Although we do not know the precise form of $G^{0}\left(\mathbf{q}, \omega_{0}\right)$, we know that $G^{0}(\mathbf{q}, \omega) \propto-1 / t$ and is a smooth function of $q$ since there are no poles at the $J=0$ limit. Within this limit, we have performed the numerical calculation for Eq. (12) and found that the band minimum is located at $(\pi, 0)$ and $(0, \pi)$.

Now let us consider the behavior of the spectral function at energy higher than the lowest pole. We can express the spectral function in the following form:

$$
A(\mathbf{k}, \omega)=\frac{\Gamma(\mathbf{k}, \omega)}{\left(\omega-E_{\mathbf{k}}\right)^{2} / R_{\mathbf{k}}^{2}+\Gamma^{2}(\mathbf{k}, \omega)},
$$

with

$\Gamma(\mathbf{k}, \omega)=z^{2} t^{2} \sum_{\mathbf{p}, \mathbf{q}} \gamma_{\mathbf{k}-\mathbf{p}}^{2} f(\mathbf{p}, \mathbf{q}) A\left(\mathbf{k}-\mathbf{q}, \omega-\omega_{\mathbf{p}}-\omega_{\mathbf{p}-\mathbf{q}}\right)$,

where $E_{\mathbf{k}}$ denotes the position of the lowest pole. Thus, for $\omega-E_{\mathbf{k}} \ll J$, we find $\Gamma(\mathbf{k}, \omega) \propto t^{2}\left(R_{\mathbf{k}} / J^{4}\right)\left(\omega-E_{\mathbf{k}}\right)^{3}$. If we extrapolate this expression to $\omega-E_{\mathrm{k}} \approx J$, we find $\Gamma\left(\mathbf{k}, E_{\mathbf{k}}+J\right) \approx t$. In this limit, the spectral function $A(\mathbf{k}, \omega) \propto 1 / t$. This result indicates that the incoherent part of the spectral function is a constant of order $1 / t$ above the pole. This picture is consistent with the previous calculations. ${ }^{6-8}$

At small doping, we can calculate the effective interaction between the quasiparticle holes by using the effective Hamiltonian (3) in the AF background. According to the above calculations, the energy spectrum of the quasiparticle holes $\varepsilon_{\mathbf{k}}(\sim J)$ has the minimum occurring at $\mathbf{k}=\mathbf{k}_{0}$. At small but finite doping, a small Fermi surface of the quasiparticles is created around $\mathbf{k}_{0}$. From the secondorder perturbation, we can obtain the effective interaction between holes mediated by the spin fluctuations

$H_{\mathrm{eff}}=\sum_{\mathbf{k}, \mathbf{k}^{\prime}} V\left(\mathbf{k}_{0}, \mathbf{k}, \mathbf{k}^{\prime}\right) C_{\mathbf{k}_{0}+\mathbf{k}_{\uparrow}}^{\dagger} C_{\mathbf{k}_{0}-\mathbf{k}_{\downarrow}}^{\dagger} C_{\mathbf{k}_{0}-\mathbf{k}^{\prime} \downarrow} C_{\mathbf{k}_{0}+\mathbf{k}^{\prime} \uparrow}$,

with

$$
\begin{aligned}
V\left(\mathbf{k}_{0}, \mathbf{k}, \mathbf{k}^{\prime}\right)=z^{2} t^{2} \sum_{\mathbf{q}} & \frac{2\left(\omega_{\mathbf{k}+\mathbf{q}}+\omega_{\mathbf{k}^{\prime}+\mathbf{q}}\right) g\left(\mathbf{k}, \mathbf{k}^{\prime}, \mathbf{q}\right)}{\left(\varepsilon_{\mathbf{k}}-\varepsilon_{\mathbf{k}^{\prime}}\right)^{2}-\left(\omega_{\mathbf{k}+\mathbf{q}}+\omega_{\mathbf{k}^{\prime}+\mathbf{q}}\right)^{2}} \\
& \times \gamma_{\mathbf{k}_{0}+\mathbf{k}+\mathbf{k}^{\prime}+\mathbf{q}} \gamma_{\mathbf{k}_{0}-\mathbf{k}-\mathbf{k}^{\prime}-\mathbf{q}},
\end{aligned}
$$

where

$$
g\left(\mathbf{k}, \mathbf{k}^{\prime}, \mathbf{q}\right)=\left(\cosh \theta_{\mathbf{k}+\mathbf{q}} \cosh \theta_{\mathbf{k}^{\prime}+\mathbf{q}}+\sinh \theta_{\mathbf{k}+\mathbf{q}} \sinh \theta_{\mathbf{k}^{\prime}+\mathbf{q}}\right)^{2} .
$$

Equations (15) and (16) have the same form as the original phonon-mediated interaction between electrons. Thus, if the interaction is attractive, the condensation of hole pairs will occur. It is interesting to note that the pair potential $V\left(\mathbf{k}_{0}, \mathbf{k}, \mathbf{k}^{\prime}\right)$ is repulsive for $\mathbf{k}_{0}=(\pi / 2, \pi / 2)$, while it is attractive for $\mathbf{k}_{0}=(\pi, 0)$ or $(0, \pi)$. This result implies that, for the finite-doping system, the holes in the corners of the pseudo-Fermi surface should have lower energy than those at $(\pi / 2, \pi / 2)$, i.e., for the system with more than one hole, the holes will favor quasiparticle states at $(\pi, 0)$ and $(0, \pi)$ momenta in agreement with the numerical simulations of small clusters with two holes. ${ }^{10,11}$ Near the Fermi surface, $\varepsilon_{\mathbf{k}} \sim \varepsilon_{\mathbf{k}^{\prime}}$, the attractive pair potential at $\mathbf{k}_{0}=(\pi, 0)$ or $(0, \pi)$ is given by

$$
V\left(\mathbf{k}, \mathbf{k}^{\prime}\right)=-2 z^{2} t^{2} \sum_{\mathbf{q}} \frac{\left(\gamma_{\mathbf{k}+\mathbf{k}+\mathbf{q}}^{\prime}\right)^{2}}{\omega_{\mathbf{k}+\mathbf{q}}+\omega_{\mathbf{k}^{\prime}+\mathbf{q}}} g\left(\mathbf{k}, \mathbf{k}^{\prime}, \mathbf{q}\right),
$$

where $\gamma_{\mathbf{k}}^{\prime}=\left(\cos k_{x}-\cos k_{y}\right) / 2$. This result suggests that the pair potential has its largest attractive component in the $d$-wave channel. So we expect that a $d$-wave pairing of the quasiparticle holes in a quantum AF background is most probable. In our analysis, the extended $s$-wave and $p$-wave states are suppressed. The reason is that with two holes having momenta $\mathbf{k}_{0}=(\pi, 0)$ or $(0, \pi)$, we cannot construct the $s$-wave and $p$-wave states with zero total momentum. Recently, Shimahara, Misawa, and Taka$\mathrm{da}^{21}$ studied the possibility of superconductivity in the $t-J$ model by using the improved Hubbard-III approximation. ${ }^{22}$ They also concluded that $d$-wave superconductivity is more favorable than $s$-wave superconductivity in the $t-J$ model. But, differing from the present work, in their consideration the superconductivity is induced by the $J$ term in Eq. (1).

Since the system undergoes $d$-wave superconducting condensation and the coherent phenomenon of the holes involves an energy of order $\delta J$ with $\delta$ as the doping concentration, we expect the transition temperature $T_{c}$ will be of order $\delta J$, which brings the $T_{c}$ scale much closer to the experimentally observed transition temperature.

In the above calculation for the pair potential, the effect of the holes on the spin background beyond the mean-field theory has been neglected. It is well known that doping will destroy the AF Néel order, which also destroys the present mechanism for pairing. However, for the small doping, if the Néel state or the short-range Néel state with large coherent length still persists, the present mechanism should survive.

In conclusion, we have presented an effective Hamiltonian to study hole dynamics and the interaction in a doped 2D quantum antiferromagnet. The advantage of our approach is that one can study the property of holes directly from the true particle operators. This is quite different from the slave-boson and the slave-fermion methods. The spectral function of holes based on our effective Hamiltonian approach is investigated and we found a quasiparticle band with a quasiparticle residue of order $J / t$ and a bandwidth of order $J$. Above the quasiparticle band is an incoherent band whose width is of order $t$. An attractive pair potential in the $d$-wave channel has also been obtained. This result suggests that the $2 \mathrm{D}$ $t-J$ model by itself could lead to $d$-wave superconductivity provided that the approximations we made within the effective Hamiltonian are sufficiently accurate for the Hubbard model in the large- $U$ limit.

We would like to thank Dr. Z. Y. Weng for useful discussions. This work is supported by a grant from the Robert A. Welch Foundation and the Texas Center for Superconductivity at the University of Houston under the Prime Grant No. MDA-972-88G-0002 from the Defense Advanced Research Project Agent and the State of Texas. T.K.L. also wishes to acknowledge partial support of this work from the Thomas F. Jeffress and Kate Miller Jeffress Memorial Trust. 
${ }^{1}$ P. W. Anderson, Science 235, 1196 (1987).

${ }^{2}$ C. Gros, R. Joynt, and T. M. Rice, Phys. Rev. B 36, 381 (1987).

${ }^{3}$ Elbio Dagotto, Adriana Moreo, Robert Joynt, Silvia Bacci, and Eduardo Gagliano, Phys. Rev. B 41, 2585 (1990).

${ }^{4}$ E. Dagotto, R. Joynt, A. Moreo, S. Bacci, and E. Gagliano, Phys. Rev. B 41, 9049 (1990).

${ }^{5}$ S. A. Trugman, Phys. Rev. B 37, 1597 (1988); 41, 892 (1990).

${ }^{6}$ C. L. Kane, P. A. Lee, and N. Read, Phys. Rev. B 39, 6880 (1989).

${ }^{7}$ S. Schmitt-Rink, C. M. Varma, and A. E. Ruchenstein, Phys. Rev. Lett. 60, 2793 (1988).

${ }^{8}$ C. Gros and M. D. Johnston, Phys. Rev. B 40, 9423 (1989).

${ }^{9}$ A. Moreo, D. J. Scalapino, R. L. Sugar, S. R. White, and N. E. Bickers, Phys. Rev. B 41, 2313 (1990).

10 J. Bonča, P. Prelovšek, and I. Sega, Phys. Rev. B 39, 7074 (1989).

${ }^{11}$ E. Dagotto, J. Riera, and A. P. Young, Phys. Rev. B 42, 2347
(1990)

${ }^{12}$ D. Poilblanc and E. Dagotto, Phys. Rev. B 42, 4861 (1990).

${ }^{13}$ M. Takahashi, Phys. Rev. B 40, 2494 (1989).

14 J. E. Hirsch and S. Tang, Phys. Rev. B 40, 4769 (1989).

${ }^{15}$ A. Auerbach and D. P. Arovas, Phys. Rev. Lett. 61, 617 (1988).

${ }^{16}$ D. P. Arovas and A. Auerbach, Phys. Rev. B 38, 316 (1988).

${ }^{17}$ Y. Okabe and M. Kikuchi, J. Phys. Soc. Jpn. 57, 4351 (1988).

${ }^{18}$ S. Chakravarty, B. I. Halperin, and D. R. Nelson, Phys. Rev. B 39, 2344 (1989).

${ }^{19}$ G. Kotliar and J. Liu, Phys. Rev. B 38, 5142 (1988).

${ }^{20}$ C. Jayaprakash, H. R. Krishnamurthy, and S. Sarker, Phys. Rev. B 40, 2610 (1989).

${ }^{21}$ H. Shimahara, S. Misawa, and S. Takada, J. Phys. Soc. Jpn. 58, 4168 (1989).

${ }^{22}$ J. Hubbard, Proc. R. Soc. London Ser. A 276, 283 (1963); 281, 401 (1964). 